\title{
Duplicated transverse cervical nerve and external jugular vein
}

\author{
Nicholas O. Gerard III ${ }^{1}$, R. Shane Tubbs ${ }^{1,2,3,4,5,6}$, Joe Iwanaga ${ }^{1,2,7,8}$ \\ ${ }^{1}$ Department of Neurosurgery, Tulane Center for Clinical Neurosciences, Tulane University School of Medicine, New Orleans, LA, ${ }^{2}$ Department of \\ Neurology, Tulane Center for Clinical Neurosciences, Tulane University School of Medicine, New Orleans, LA, USA, ${ }^{3}$ Department of Anatomical \\ Sciences, St. George's University, St. George's, Grenada, ${ }^{4}$ Department of Structural \& Cellular Biology, Tulane University School of Medicine, New \\ Orleans, LA, ${ }^{5}$ Department of Neurosurgery and Ochsner Neuroscience Institute, Ochsner Health System, New Orleans, LA, ${ }^{6}$ Department of Surgery, \\ Tulane University School of Medicine, New Orleans, LA, USA, ${ }^{7}$ Dental and Oral Medical Center, Kurume University School of Medicine, Kurume, \\ Fukuoka, ${ }^{8}$ Division of Gross and Clinical Anatomy, Department of Anatomy, Kurume University School of Medicine, Kurume, Fukuoka, Japan
}

\begin{abstract}
The transverse cervical nerve arises from anterior rami of the second and third cervical spinal nerves via the cervical plexus. We present a case of a left duplicated transverse cervical nerve with a duplicated external jugular vein in a 72-year-old female cadver. The transverse cervical nerve bifurcated into two branches, i.e., superficial and deep branches, lateral to the sternocleidomastoid muscle. The superficial branch ran lateral to the duplicated external jugular vein and gave a cutaneous branch to the area below the great auricular nerve and cutaneous branches to the skin of the neck. The deep branch ran medial to the duplicated external jugular vein, joined the anterior branch of the superficial transverse cervical nerve and cervical branch of the facial nerve, and terminated into the skin. This case adds to the growing data on individual variability that should be considered when operating on the anterolateral neck.
\end{abstract}

Key words: Transverse cervical nerve, External jugular vein, Anatomy, Cadaver, Neck surgery

Received April 13, 2021; Revised April 27, 2021; Accepted April 29, 2021

\section{Introduction}

The transverse cervical nerve (TCN) arises from the cervical plexus, more specifically derives from anterior rami of the second and third cervical spinal nerves (C2-3). The TCN innervates the cutaneous skin covering the anterolateral cervical region, it can be located upon dissection using the sternocleidomastoid muscle (SCM) as a landmark. Typically, the TCN courses posterior to anterior around the middle of the muscle passing just deep to the external jugular vein [1]. After piercing the cervical fascia, at Erb's point, the TCN splits into a superior branch anastomosing with the facial nerve,

\footnotetext{
Corresponding author:

Joe Iwanaga (i)

Department of Neurosurgery, Tulane Center for Clinical Neurosciences, Tulane University School of Medicine, New Orleans, LA 70112, USA E-mail: iwanagajoeca@gmail.com
}

and the inferior branch innervating the cutaneous skin of the neck [2]. Anatomic variations in these neural structures are important to consider when operating in the anterolateral aspect of the neck. Here we present a caseof a left duplicated TCN in a 72-year-old female donor. We will review the dimensions, dissection technique, and clinical implications of variant TCNs. To our knowledge, this is the first reported case of a duplicated TCN.

\section{Case Report}

During a routine dissection of a 72-year-old European descendent female cadaveric head, a rare anatomical variation of the TCN was noted on the left neck (Fig. 1). The TCN originated from anterior rami of $\mathrm{C} 2$ and $\mathrm{C} 3$ spinal nerves and had a common trunk with a great auricular nerve (GAN). The GAN presented a normal morphology that ascended to the ear. The TCN bifurcated into two branches, i.e., super- 


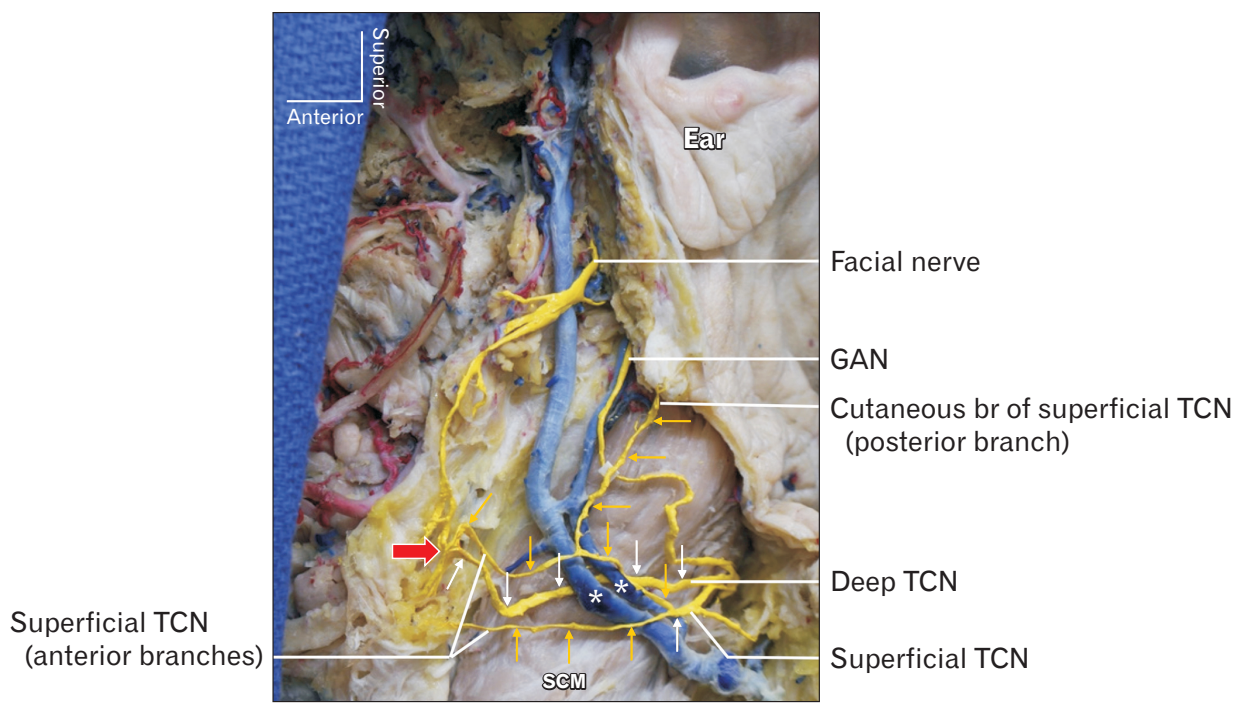

Fig. 1. Duplication of the TCN and external jugular vein (asterisk). A small branch of the cervical branch of the facial nerve, one of the anterior branch of the superficial TCN and deep TCN unite and innervate the skin (large red arrow). Small orange arrows indicate superficial branches of TCN; small white arrows indicate deep branch of TCN. GAN, great auricular nerve; SCM, sternocleidomastoid muscle; TCN, transverse cervical nerve. ficial and deep branches, lateral to the SCM. The superficial branches (orange arrows in Fig. 1) ran lateral to the duplicated external jugular vein and gave a cutaneous branch to the area below the GAN (posterior branch) and cutaneous branch to the skin of the neck (anterior branches). The deep branch (white arrows in Fig. 1) ran medial to the duplicated EJV, joined one of the anterior branches of the superficial TCN and a branch of the cervical branch of facial nerve, and terminated into the skin. No surgical scars or other variations were found in the dissected area or on the right side.

\section{Discussion}

Both GAN and TCN are sensory fibers that originate from the anterior rami of the $\mathrm{C} 2$ and $\mathrm{C} 3$. The TCN in this report innervated skin of the anterolateral neck (anterior branches) and skin below the ear (posterior branch). Anterior branches from superficial TCN and deep TCN might be considered normal TCN, and the posterior branch might be a part of the GAN in terms of area of innervation.

Relationship of the duplicated external jugular veins and superficial and deep TCNs are noted in the present case. The anterior external jugular vein is considered a remnant of the anterior communication between the external jugular vein and facial vein [3]. Failure of the degeneration of the anterior communication might have affected the positional relationship.

Anastomotic connections between the TCN and branches of cranial nerve VII, the facial nerve, are well described [4]. The facial nerve has more anastomotic neural connections than any other cranial nerve. The superficial branch of TCN typically joins the cervical branch of the facial nerve. However, authors have recently identified communication between the TCN and the marginal mandibular branch of the facial nerve [5]. The prevalence of communication between the TCN and the marginal mandibular branch of the facial nerve is $2.1 \%$ in cadaveric studies [6]. Clinically, this anastomotic connection can be implicated in dental anesthesia, possibly explaining inter-patient variability of outcome of the inferior alveolar nerve blocks [7].

Upon dissection, the TCN is often sacrificed during surgical procedures on the neck such as a radical neck dissection, carotid endarterectomy and cervical lymph node dissections. There are potential risks for damage to the TCN that extend further than loss of cutaneous sensory innervation of the neck. The crosstalk between the TCN and cranial nerves is sufficient evidence for further anatomical evaluations. Carotid endarterectomy complications include damage to the cranial nerves $(3.8 \%)$ of the time in a retrospective analysis, of which (0.8\%) affected the marginal mandibular branch of the facial nerve, $(0.8 \%)$ laryngeal nerve, $(1.2 \%)$ accessory nerve, and (2.5\%) hypoglossal nerve [8].

Anatomic variants in the superficial venous vasculature are common in the head and neck. In this donor, along with a duplicated TCN, a duplicated external jugular vein was identified. This variation has been reported in twenty sides across 16 cases in the literature [3]. The highly variable anatomy common to this region is important for head and neck reconstructive surgery. Surgeons operating on the anterior branches of the external carotid artery must take care with 
these variations when grafting vessels to the neck [9].

In conclusions, This duplicated TCN adds to the growing data on individual variability that should be considered when operating on the anterolateral neck.

\section{ORCID}

Nicholas O. Gerard III:

https://orcid.org/0000-0002-1690-443X

R. Shane Tubbs: https://orcid.org/0000-0003-1317-1047

Joe Iwanaga: https://orcid.org/0000-0002-8502-7952

\section{Author Contributions}

Conceptualization: RST, JI. Data acquisition: RST, JI. Data analysis or interpretation: NOG. Drafting of the manuscript: NOG. Critical revision of the manuscript: RST, JI. Approval of the final version of the manuscript: all authors.

\section{Conflicts of Interest}

No potential conflict of interest relevant to this article was reported.

\section{Acknowledgements}

The authors sincerely thank those who donated their bodies to science so that anatomical research could be performed. Results from such research can potentially increase mankind's overall knowledge that can then improve patient care. Therefore, these donors and their families deserve our highest gratitude [10].

\section{References}

1. Tubbs RS, Rizk E, Shoja MM, Loukas M, Barbaro NM, Spinner RJ. Nerves and nerve injuries: vol 1: history, embryology, anatomy, imaging, and diagnostics. London: Academic Press; 2015. p. 441-9.

2. Erb W. Handbook of electro-therapeutics. New York: William Wood and Company; 1883. p. 122-4

3. Ono K, Yoshioka N, Hage D, Ibaragi S, Tubbs RS, Iwanaga J. Duplication of the external jugular vein: a language barrier of database search in classic anatomical studies. Surg Radiol Anat 2021 Feb 23 [Epub]. https://doi.org/10.1007/s00276-021-027176.

4. Domet MA, Connor NP, Heisey DM, Hartig GK. Anastomoses between the cervical branch of the facial nerve and the transverse cervical cutaneous nerve. Am J Otolaryngol 2005;26:16871.

5. Reuther WJ, Blythe JN, Anand R, Brennan PA. Communication of the transverse cervical nerve with the marginal mandibular nerve: a previously unreported anatomical variant. $\mathrm{Br} \mathrm{J}$ Oral Maxillofac Surg 2014;52:577-8.

6. Brennan PA, Mak J, Massetti K, Parry DA. Communication between the transverse cervical nerve $(\mathrm{C} 2,3)$ and marginal mandibular branch of the facial nerve: a cadaveric and clinical study. Br J Oral Maxillofac Surg 2019;57:232-5.

7. Lin K, Uzbelger Feldman D, Barbe MF. Transverse cervical nerve: implications for dental anesthesia. Clin Anat 2013;26:688-92.

8. Beasley WD, Gibbons CP. Cranial nerve injuries and the retrojugular approach in carotid endarterectomy. Ann R Coll Surg Engl 2008;90:685-8.

9. Tan BK, Wong $\mathrm{CH}$, Chen HC. Anatomic variations in head and neck reconstruction. Semin Plast Surg 2010;24:155-70.

10. Iwanaga J, Singh V, Ohtsuka A, Hwang Y, Kim HJ, Moryś J, Ravi KS, Ribatti D, Trainor PA, Sañudo JR, Apaydin N, Şengül G, Albertine KH, Walocha JA, Loukas M, Duparc F, Paulsen F, Del Sol M, Adds P, Hegazy A, Tubbs RS. Acknowledging the use of human cadaveric tissues in research papers: recommendations from anatomical journal editors. Clin Anat 2021;34:24. 